\title{
Frequency-selective plasmonic wave propagation through the overmoded waveguide with photonic-band-gap slab arrays
}

\author{
Young-Min Shin ${ }^{\text {a) }}$ \\ Department of Physics, Northern Illinois University, Dekalb, Illinois 60115, USA and Accelerator Physics \\ Center (APC), Fermi National Accelerator Laboratory (FNAL), Batavia, Illinois 60510, USA
}

\begin{abstract}
Confined propagation of guided waves through the periodically corrugated channel sandwiched between two staggered dielectric photonic-band-gap slab arrays is investigated with the bandresponse analysis. Numerical simulations show that longitudinally polarized evanescent waves within the band gap propagate with insertion loss of $\sim-0.2$ to $1 \mathrm{~dB}(-0.05$ to $0.4 \mathrm{~dB} / \mathrm{mm}$ at G-band) in the hybrid band filter. This structure significantly suppresses low energy modes and higher-order-modes beyond the band-gap, including background noises, down to $\sim-45 \mathrm{~dB}$. This would enable the single-mode propagation in the heavily over-moded waveguide (TEM-type), minimizing abnormal excitation probability of trapped modes. This band filter could be integrated with active and passive RF components for electron beam and optoelectronic devices.
\end{abstract}

Periodic conducting structures have been utilized as active slow wave media interacting with high energy electron beams in a wide range of applications from high gradient linear accelerators to coherent power radiation sources. The distorted dispersion relation of metallic lattices constitutes effective plasmonic modes with negative permittivity (epsilon-negative materials, $\varepsilon<0) .{ }^{1-4}$ The non-radiative waves have been widely used in microwave regimes as they can be readily guided in passive components or strongly excited by energy-momentum-coupling with kinetic electrons as an active medium. ${ }^{5}$ The confined evanescent waves induce electron bunches when the current density exceeds thermo-dynamic divergence $\left(\propto f^{-2}\right){ }^{6}$ This gives rise to a strong coherent electron-photon interaction that is commonly used for high intensity photon generation or high gradient $\mathrm{RF}$ acceleration. The spectral regime of the electronic RF devices has rapidly increased from microwave to nearterahertz (near-THz) wave regime $(0.1-1 \mathrm{THz})$ as device size can be significantly reduced proportional to the wavelength. Energy conversion efficiency on the photon-emission or -absorption process is thus noticeably reduced as the optical cycle is decreased. ${ }^{7,8}$ Recent trends in the development of $\mathrm{THz}$ electron beam devices have gradually transitioned to elliptical sheet beams from round ones as enlarging the beam width either increases radiation intensity or reduces beam current loading. Among various planar slow wave structures (SWSs), ${ }^{9-19}$ recently micro-channels with an asymmetrically aligned double grating array ${ }^{20-22}$ were intensively studied for frequency-tunable coherent radiation source application as they have large instantaneous bandwidth of plasmonic modes that are strongly confined in the beam channel with small ohmic losses. However, this broad band characteristic can lead to unstable oscillation of abnormal modes, which possibly destabilize beam-wave interactions, and perturb

\footnotetext{
${ }^{\text {a) }}$ Author to whom correspondence should be addressed. Electronic mail: alcolpeter@gmail.com.
}

normal electronic energy conversion. In particular, the structure becomes heavily overmoded (TE $\rightarrow$ TEM) as its aspect ratio between $x-y$ transverse dimensions is increased to expand the beam-wave interactive area. It is thus certain that there is a limitation in increasing the aspect ratio of the sheet beam structure due to low-energy mode excitation. A substantial solution to the issue could be obtained from bandgap structures. This paper will present the band-selective planar waveguide combined with the photonic crystal slabs. The composite structure permits only non-radiative photonic-band-gap $(\mathrm{PBG})$ modes $^{23,24}$ to propagate through the sub-wavelength micro-channel. Comparative numerical analysis on the spectral response and field distribution will be discussed in detail.

In principle, the lowest frequency (cutoff) of a rectangular TE-mode waveguide is determined by waveguide width, so TE modes become closer to TEM modes as the cutoff decreases. Figure 1 shows the 0th order dispersion curves $(m=0)$ of TE- and TEM-waveguides. Normalized structural dimensions of the designed models are specified with $a_{0} / d_{0}$ $=0.75, L_{0} / d_{0}=0.6, b_{0} / d_{0}=0.33$, and $h_{0} / d_{0}=1.674$, where $d_{0}$ is the grating period of a unit cell. The surface wall conductivity is defined with oxygen-free high-conductivity (OFHC) copper $\left(\sigma=5.8 \times 10^{8}\left[\Omega^{-1} \mathrm{~m}^{-1}\right]\right)$. With an increase in the guide width, the entire eigenfrequencies of the fundamental band (grey solid $\rightarrow$ grey dashed) are lowered as the other dimensions remain consistent with the ones of the TEmode waveguide. In the 2nd TEM waveguide (grey dashed line), the grating height $\left(L_{0}\right)$ is shortened to keep the upper cutoff (stop-band) at the same frequency as the TM mode dispersion curve, which further extends the $x-y$ dimensional aspect ratio. The passband of the 2nd TEM SWS (higher aspect ratio: black solid) thus tends to become even more heavily overmoded with larger phase and group velocities. As aforementioned, spatial harmonic components of lower frequency modes are readily coupled with low energy electrons, so that while haunting around in the structure, once excited, 

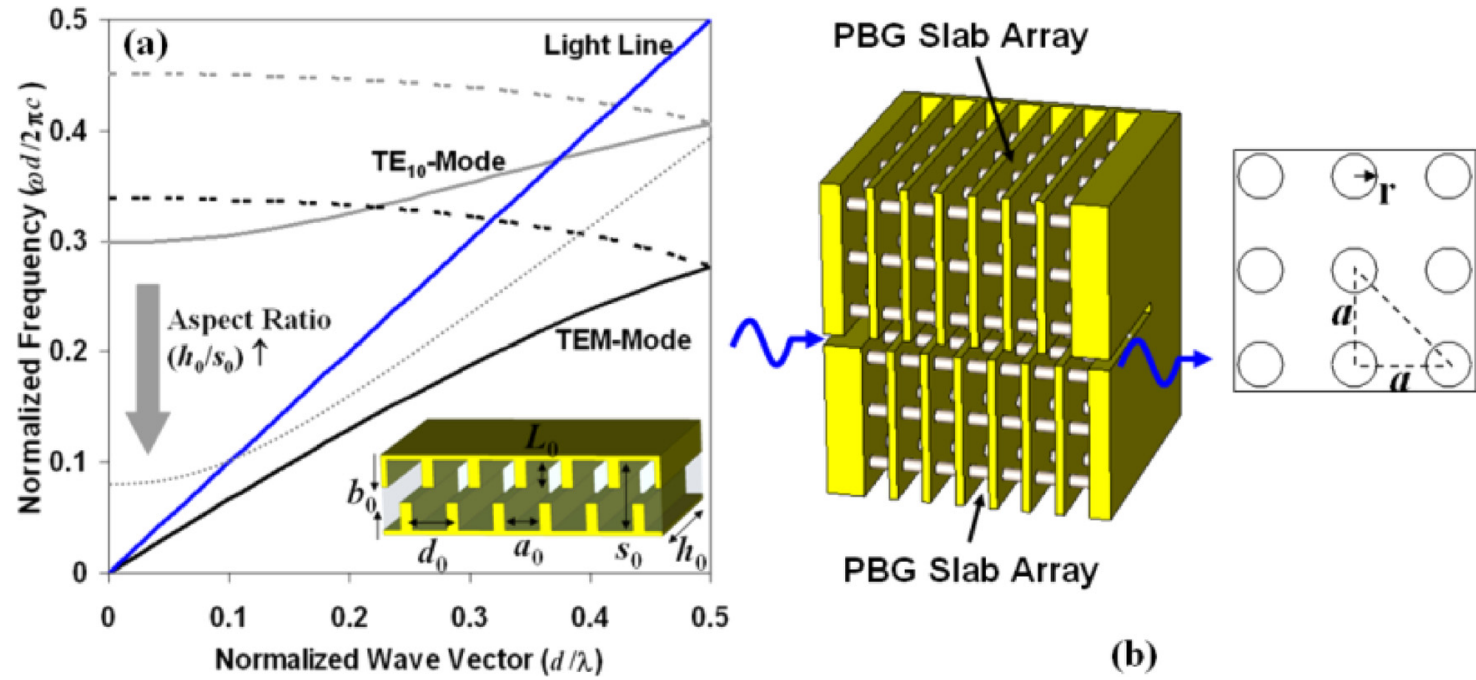

(b)

FIG. 1. (a) Dispersion graph of the overmoded planar SWS. Inset is the schematic drawing of the staggered double grating arrayed waveguide with the dimensional parameters (TEM-mode). (b) 3D drawing of the PBG-slab-arrayed waveguide and unit cell of the designed PBG slab.

they are often self-amplified by phase-mismatching condition, which can cause strong perturbation to electron-photon coupling.

Embodiment of PBG elements in guided wave structures significantly reduces background noise level, including nonPBG modes, in the dynamic spectral range. In Fig. 1(b), the top and bottom gratings are replaced with the arrayed $\mathrm{PBG}$ slabs, which consist of alumina $\left(\mathrm{Al}_{2} \mathrm{O}_{3}: \varepsilon_{\mathrm{r}}=9.4, \tan \delta=\right.$ $0.0004)$ rods. In the crystal designed with the lattice constant of $a / \lambda \sim 0.3$ and filling ratio of $r / a \sim 0.25$, normalized global band-gap emerges at $\mathrm{f}\left(=\omega d_{0} / 2 \pi c\right)=0.25-0.3$. As depicted in Fig. 1(b), like an ordinary confined slow wave structure, electromagnetic (EM) waves bouncing back from the crystal lattices in the band-gap spectra that satisfy the Bragg condition propagate through the channel between the two PBG slab-arrays with strong field confinement. On the other hand,

non-resonant eigenmodes outside the band gaps are freely radiated through the lattices, which are thereby strongly suppressed in the channel. Figure 2(a) shows the 0st, 1st, and 2nd spatial harmonic dispersion curves ( $m=0,1$, and 2$)$ of the fundamental passband of the TEM-mode waveguide. An electron beam with kinetic energy up to $20 \mathrm{keV}$ encounters the 1 st and 2 nd fundamental bands at the three synchronous points that are one forward-wave $\left(d_{0} / \lambda=1-1.5\right)$ and two backward-wave modes $\left(d_{0} / \lambda=0.5-1\right.$ and $\left.1.5-2\right)$. The mixed phase-velocities lead to instantaneous excitation of several frequency components. The excited backward wave modes and reflected forward ones can be rapidly amplified due to feedback-interaction with the forward propagating beam if their gain per length exceeds attenuation of the designed structure and excessive impedance mismatches present at the end-boundaries. Excitation of the non-operational harmonic modes would disperse the beam energy over a broad spectrum, possibly diminishing radiation efficiency. Also, it could destabilize density modulation leading to an increase of local beam loading, which may cause beam breakups by a de-bunching effect. Figure 2(b) shows the transmission spectrum $\left(\mathrm{S}_{21}\right)$ of the PBG-embedded TEM-type slow wave structure (inset), which has the same plasmonic waves over the PBG that thereby enable evanescent field propagation with nearly zero insertion loss $(=-0.2$ to $1 \mathrm{~dB})$ at $\mathrm{f}=$ $0.136-0.194$ corresponding to attenuation of $0.05-0.4$ $\mathrm{dB} / \mathrm{mm}$ (G-band). Note that the modes out of the band gap are highly lossy and radiative, so they get annihilated as the waveguide is prolonged. The insertion losses below the PBG ( $<0.136$ ) thus rapidly increase with an increase in the waveguide length. As depicted in Fig. 1, it is evident that

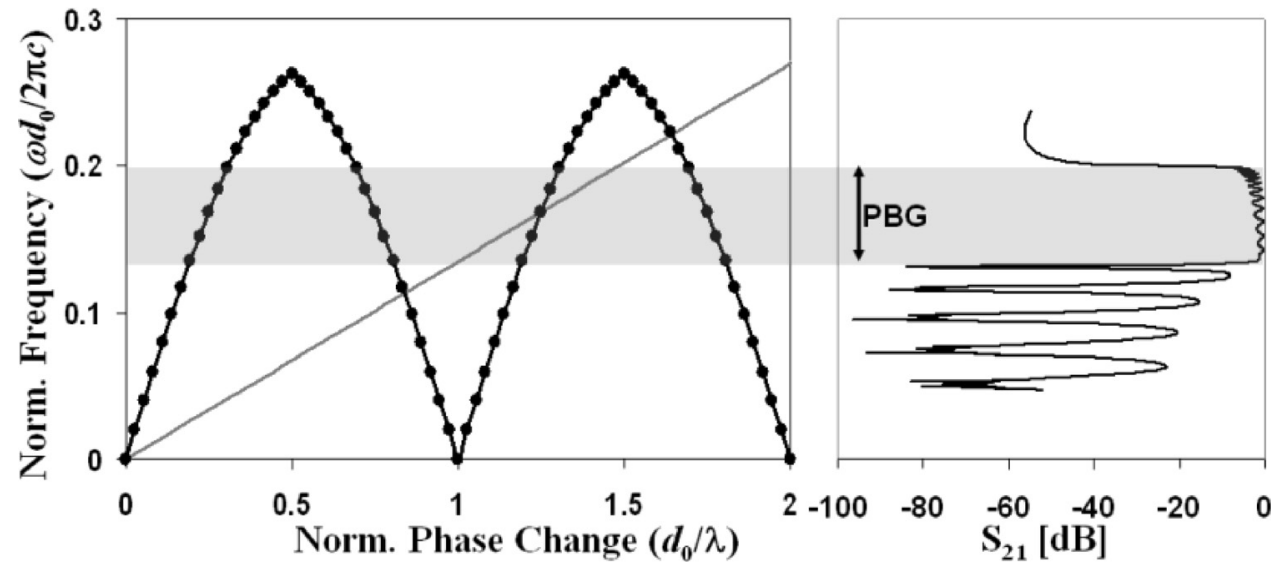

FIG. 2. (a) 0th, 1st, and 2nd order dispersion curves $(m=0,1$, and 2$)$ of TEM-mode SWS with the beam line. (b) Transmission spectrum (S21 graph) of the PBG-slab arrayed SWS. 

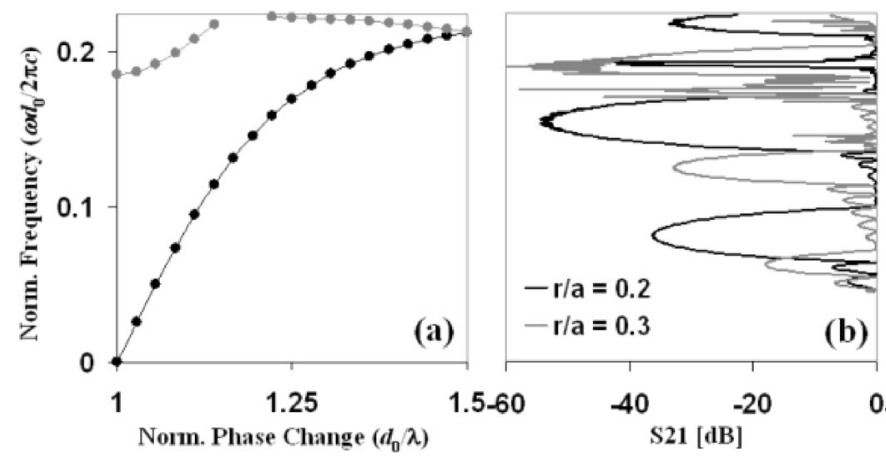

only the non-radiative plasmon modes in the band gap synchronously couple with electrons if the kinetic energy satisfies the coupling condition, $\gamma=\omega /\left.\omega c k_{s p}\right|_{m=1}$, of the PBG modes (gray-shaded area), where $\gamma$ is the relativistic factor, $\gamma=\sqrt{1-(1+\sigma)^{-2}}$, and $\sigma=V_{\mathrm{e}} / V_{\mathrm{n}}\left(V_{\mathrm{n}}=m_{\mathrm{e}} c^{2} / e=\right.$ $\left.5.11 \times 10^{5}[\mathrm{~V}]\right)$. The two other synchronous points $(m=1$ and $m=2$ ) out of the band gap are inactive modes that have excessively higher radiating losses than energy gain per length. Figure 3 shows dispersion curves, (a), of the TEM waveguide with the staggered vane arrays and transmission spectra of the photonic crystal slab, (b), and the PBG-slab embedded TEM waveguide, (c), with respect to two filing ratios, $\mathrm{r} / a=0.2$ and 0.3 . In Fig. 3(b), the crystal slab arrays with $\mathrm{r} / a=0.2$ and 0.3 have three band gaps below the coalesced mode (upper cutoff) of the fundamental passband (a). Therefore, only the eigenmodes in the band gaps are allowed to propagate through the beam channel. Figure 3(c) shows an apparent emergence of three spectral bands with zero insertion loss which are exactly matched with the band gaps in Fig. 3(b). The background loss level of radiative non-PBG modes is about $-45 \mathrm{~dB}$ that is low so as not to cause an abnormal beam-wave interaction. In Figs. 3(b) and 3(c), it is readily noticeable that the band-gap widths of $\mathrm{r} / a=0.3$ is only about half of widths of $\mathrm{r} / \mathrm{a}=0.2$, which implies that increase of the rod size relative to the lattice constant leads to raising population density of guided photonic eigenstates with narrowing their intrinsic bandwidths. This indicates that the active spectral range and dispersion characteristics of confined waves are primarily determined by a lattice constant and a filling ratio of the photonic crystal filter rather than by the grating dimensions.

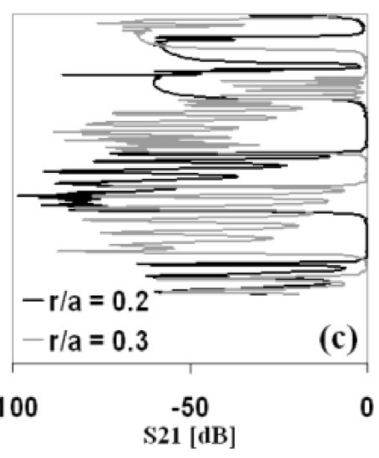

FIG. 3. (a) 0th order dispersion graph of TEM-mode SWS and transmission spectra of the PBG-slabs (b), and the PBGslab-arrayed SWS (c), of $r / a=0.2$ (black) and 0.3 (grey).

The simulation models in Figures 2 and 3 are designed to be the ideal TEM-mode waveguide with the magnetic boundaries in the major transverse axis that appear impractical in actual application. The actual device model is thus designed with the oversized TE-mode waveguide that has an electric boundary. As depicted in Fig. 4, the designed waveguide is so wide as to be heavily overmoded with normalized cutoff frequency $\mathrm{f}_{\mathrm{c}}\left(=\omega_{\mathrm{c}} a / 2 \pi c\right)$ of 0.105 . In this model, both lateral sides of the PBG-slabs are completely covered by the metal plates, so that eigenmodes exist only above the lower cutoff of the oversized waveguide. Figure 4(a) is the dispersion curves of the two slow waveguide structures embedded with the grating arrays (grey) and the photonic crystal slab arrays (red) that are procured by the finite-integral-technique (FIT) eigenmode solver. ${ }^{25}$ The blue-shaded and whitecolored areas represent the photonic zone (far-field, $k<2 \pi / \lambda$ ) and plasmonic one (near-field, $k>2 \pi / \lambda$ ), respectively. The plotted points indicate all scattering modes corresponding to a phase change per period of the staggered corrugation. These unguided waves travel around and radiate in free space, and therefore have no interaction with the electron beam. In Fig. 4(b), the global band-gap noticeably emerges from $f=0.265$ to $f=0.31$ at either the photonic or plasmonic wave area. There is no other photon mode than interactive plasmon modes in the first forbidden band. The channel confined interaction modes disappear beyond the stop-band as they radiate to annihilate owing to the nonresonant matching condition with the crystal lattices. Note that the PBG-modes are considerably more dispersive than fundamental eigenmodes of the oversized waveguide (greyline). The fringe evanescent fields around the boundaries between the waveguide and the PBG-slabs distort the

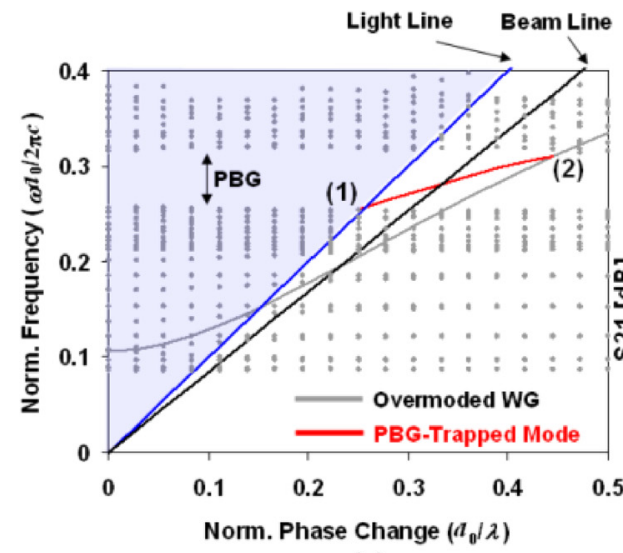

(a)

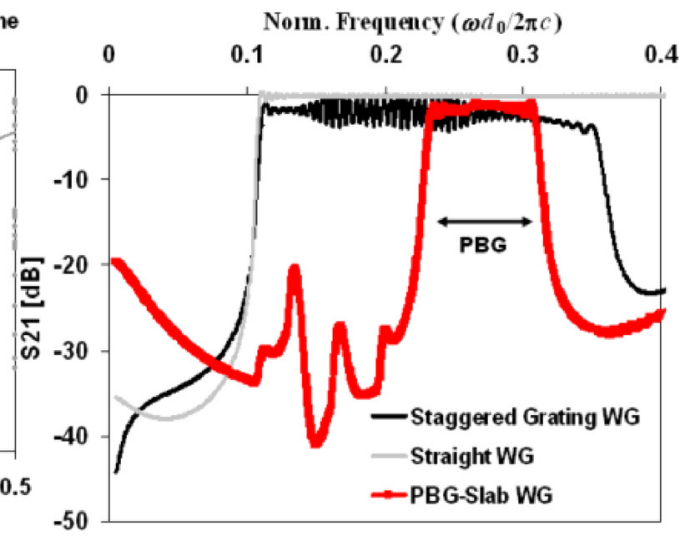

(b)
FIG. 4. (a) 0th order dispersion graphs (band-diagram) of the PBG-slab-arrayed SWS and TEM-mode SWS. (b) Transmission spectra (S21) of staggered grating waveguide, straight waveguide, and PBG-slab-arrayed waveguide. 


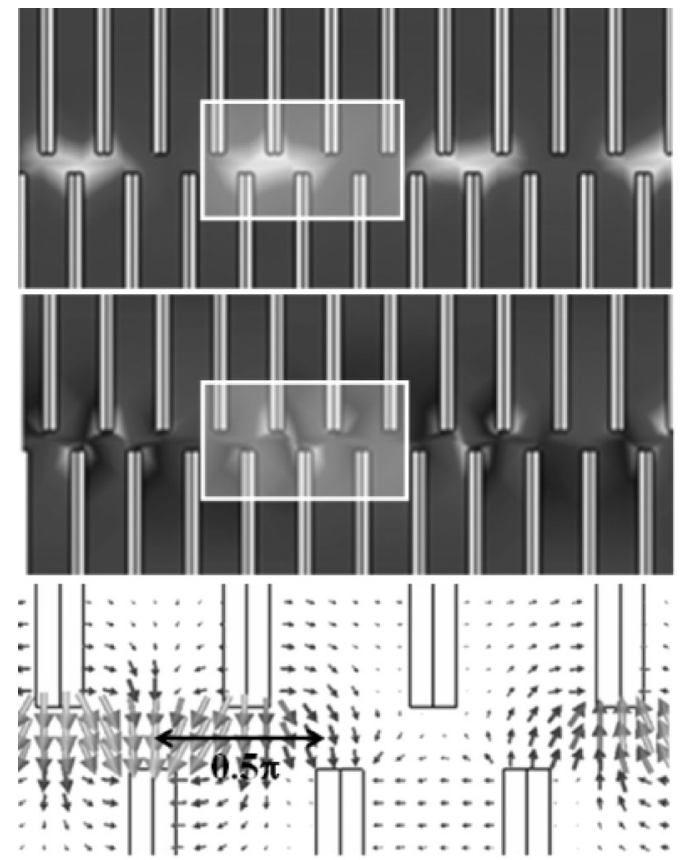

(a)
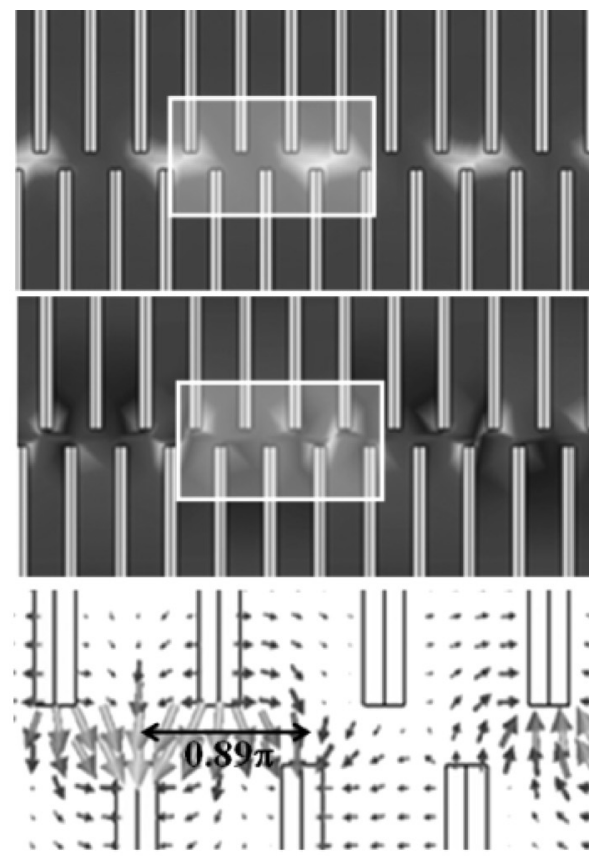

(b)
FIG. 5. Two dimensional spatial distri-

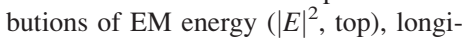
tudinal electric field $\left(E_{\mathrm{z}}\right.$, middle) and vector field ( $\vec{E}$, bottom) of (a) lowest frequency mode ((1): $0.5 \pi$-mode) and (b) highest frequency mode ((2): $0.89 \pi$ mode) in Fig. 4(a). dispersion relation of the fundamental passband. These highly dispersive modes thus have larger group velocities with higher interaction impedances compared to the traveling-wave modes. Therefore, as the PBG-waveguide supports only propagation of the dispersive waves within the band-gap, the electron beam purely interacts with the active modes of $k\left(=d_{0} / \lambda\right)=0.25-0.45$ and $\mathrm{f}=0.265-0.31$ without exciting noisy wakefields. Figure 4(b) exhibits transmission graphs $\left(\mathrm{S}_{21}\right)$ of three types of the slow wave structures: straight waveguide, staggered double grating waveguide, and PBG-slab embedded slow waveguide. $\mathrm{TE}_{10}$ waves above the cutoff of the rectangular waveguide pass through the straight waveguide, with almost no insertion loss. The fundamental passband of the corrugated waveguide, on the other hand, ends at $\mathrm{f}=0.353$, which corresponds to $\sim 100 \%$ instantaneous bandwidth and -0.17 to $3.6 \mathrm{~dB}$ insertion loss. Corrugation of the wave traveling path degenerates energy states of confined modes due to their momentum divergence at the upper cutoff, where localized fields longitudinally, rather than laterally, resonant with the periodic structure. In Fig. 4(b), the $S_{21}$ spectra clearly depict that only the PBG-modes dominantly travel in the sub-wavelength channel with nearly zero insertion loss, whereas all other modes beyond the band gap are completely suppressed down below $-30 \mathrm{~dB}$. It is observed that the PBG still strongly appears $(<\sim-1 \mathrm{~dB})$ even with three PC layers, although it fades away if the number of layer is less than 3. Irregularly arising parasitic wakefields could thus be intrinsically ruled out from the beam-wave interaction. The sensitivity analysis on length and radius of PC rods in the aluminum plates ended up showing no noticeable change of the transmission graph. The efficient mode filtering thus remains appreciably insensitive to deviation of the structural dimensions as the corrugated slow wave channel has broad spectral coverage.

In Fig. 5, while in the channel transverse component $\left(E_{y}\right)$ dominantly appear in the electric field distributions, one can see that their field orientation is abruptly transitioned to the longitudinal direction between the two staggered vanes, accommodating sinusoidal field modulation of the axial field $\left(E_{z}\right)$, which has a constructive interaction with the electron beam with an energy gain. The quasi-optical energy localization remains effective down to $0.5 \pi(f=0.265)$ and up to $0.89 \pi$ ( $f=0.31$, whereas photonic modes rapidly lose the structural resonance as away from the band gap. It is noticeable that extended parallel conductor plates on account of the PBG slabs enhance capacitance of the resonance modes, leaking fringe fields of the longitudinal components. It may cause reduction of interaction impedances that could be compensated for by increasing the cross-sectional aspect ratio of the beam channel.

In conclusion, the staggered double PBG-slab arrays enable overmoded planar slow waveguides to induce monochromatic wave propagation of a single plasmonic mode within a narrow band-gap. Simulation analysis presented that the PBG-embedding selectively suppresses the nonresonating modes in background spectrum below -30 to 40 $\mathrm{dB}$. This highly selective band filtering noticeably improves spectral purity of plasma-interactive evanescent waves in the over-moded slow waveguides, free from the instability problems such as mode competition/conversion, cutoff/backward wave oscillation, and noise excitation in RF devices. The quasi-optical mode-filtering waveguides support efficient energy conversion, which can be utilized as a passive or active component for various optoelectronic and electron beam devices.

\footnotetext{
R. A. Shelby, D. R. Smith, and S. Schultz, Science 292(5514), 77-79 (2001)

${ }^{2}$ J. B. Pendry, Phys. Rev. Lett. 85, 3966 (2000).

${ }^{3}$ V. George and K. G. Balmain, Negative-Refraction Metamaterials: Fundamental Principles and Applications (Wiley, 2005), p. 340.

${ }^{4}$ R. W. Ziolkowski, Metamaterials: Physics and Engineering Explorations (Wiley, 2006), pp. xv, 3-30, 37, 143-150, 215-234, 240-256.
} 
${ }^{5}$ R. K. Parker, R. H. Abrams, B. G. Danly, and B. Levush, IEEE Trans. Microwave Theory Tech. 50, 835 (2002).

${ }^{6} \mathrm{~K}$. Mizuno and S. Ono, "Comment on 'Traveling wave oscillations in the optical region: A theoretical examination," J. Appl. Phys. 46(4), 1849 (1975).

${ }^{7}$ X.-C. Zhang, Phys. Med. Biol. 47, 3667 (2002).

${ }^{8}$ C. Sirtori, "Applied physics: Bridge for the terahertz gap," Nature (London) 417, 132-132 (2002).

${ }^{9}$ B. E. Carlsten, S. J. Russell, L. M. Earley, W. B. Haynes, F. Krawczyk, E. Smirnova, Z.-F. Wang, J. M. Potter, P. Ferguson, and S. Humphries, in Proceedings of the Seventh Workshop on High Energy Density and High Power RF, 2006, edited by D. K. Abe and G. S. Nusinovich (American Institute of Physics, New York, 2006), Vol. 807, p. 326.

${ }^{10}$ K. T. Nguyen, J. Pasour, E. L. Wright, D. E. Pershing, and B. Levush, in Proceedings of the 10th International Vacuum Electronics Conference, Rome (IEEE, New York, 2009), p. 298.

${ }^{11}$ P. B. Larsen, D. K. Abe, S. J. Cooke, B. Levush, T. M. Antonsen, and R. E. Myers, in Proceedings of the 10th International Vacuum Electronics Conference (IEEE, New York, 2009), p. 225.

${ }^{12}$ A. G. Bailey, E. I. Smirnova, L. M. Earley, B. E. Carlsten, and J. L. Maxwell, Proc. SPIE 6120, 14 (2006).

${ }^{13}$ S. Humphries, S. Russell, B. Carlsten, L. Earley, and P. Ferguson, Phys. Rev. ST Accel. Beams 7, 060401 (2004).

${ }^{14}$ S. K. Wong, J. S. Kim, J. D. Goldberg, D. H. Edgell, and W. L. Spence, in Proceedings of the 18th Particle Accelerator Conference, New York, 1999 (IEEE, New York, 1999), p. 1043.
${ }^{15}$ S. J. Russell, Z. F. Wang, W. B. Haynes, R. M. Wheat, B. E. Carlsten, L. M. Earley, S. Humphries, and P. Ferguson, Phys. Rev. ST Accel. Beams 8, 080401 (2005).

${ }^{16}$ M. A. Basten, J. H. Booske, and J. Anderson, IEEE Trans. Plasma Sci. 22, 960 (1994).

${ }^{17}$ B. E. Carlsten, S. J. Russell, L. M. Earley, F. L. Krawczyk, J. M. Potter, P. Ferguson, and S. Humphries, IEEE Trans. Plasma Sci. 33, 85 (2005).

${ }^{18}$ M. E. Read, G. Miram, R. L. Ives, V. Ivanov, and A. Krasnykh, in Proceedings of the 22nd Particle Accelerator Conference, 2003, edited by J. Chew, P. Lucas, and S. Webber (IEEE, New York, 2003), p. 1137.

${ }^{19}$ J. H. Booske, M. A. Basten, and A. H. Kumbasar, Phys. Plasmas 1, 1714 (1994).

${ }^{20}$ L. R. Barnett and Y. M. Shin, "Barnett-shin traveling-wave tube (TWT) slow wave circuit for millimeter and sub-millimeter wave vacuum electronic microwave amplifiers and oscillators," U.S. patent 60979392 (12 October 2007).

${ }^{21}$ Y.-M. Shin and L. R. Barnett, Appl. Phys. Lett. 92, 091501 (2008).

${ }^{22}$ Y.-M. Shin, L. R. Barnett, and N. C. Luhmann Jr., Appl. Phys. Lett. 93, 221504 (2008)

${ }^{23}$ E. I. Smirnova, C. Chen, M. A. Shapiro, J. R. Sirigiri, and R. J. Temkin, "Simulation of photonic band gaps in metal rod lattices for microwave applications," J. Appl. Phys. 91, 960 (2002).

${ }^{24}$ E. I. Smirnova, A. S. Kesar, I. Mastovsky, M. A. Shapiro, and R. J. Temkin, "Demonstration of a 17-GHz, high-gradient accelerator with a photonic-band-gap structure," Phys. Rev. Lett. 95, 074801 (2005).

${ }^{25}$ CST Microwave Studio (Studio Suite 2011) Release Version 2011.07. 